\title{
Feeding difficulties: etiology and growth parameters
}

Katarzyna M. Bąbik ${ }^{1,2}$, Andrea Horvath ${ }^{1}$, Piotr Dziechciarz ${ }^{1}$, Paweł Ostaszewski ${ }^{2}$

${ }^{1}$ Department of Pediatrics, Medical University of Warsaw, Poland ${ }^{2}$ SWPS University of Social Sciences and Humanities, Faculty of Psychology in Warsaw, Poland

Submitted: 29 May 2020

Accepted: 17 August 2020

Arch Med Sci

DOI: https://doi.org/10.5114/aoms.2020.100187

Copyright (@ 2020 Termedia \& Banach

\begin{abstract}
Introduction: In the first year of life it is crucial to maintain a well-balanced diet. It ensures optimal growth and development of the child. Feeding difficulties experienced by a child might lead to impairment of the nutritional status. Despite the importance of the subject, we did not find much recent published information. The aim of the study was to assess the etiology and growth parameters in children with feeding difficulties.

Material and methods: Children with feeding difficulties admitted to the Department of Pediatrics (September 2013 - April 2019) were recruited. Feeding difficulties were diagnosed by a minimum of two specialists. Based on medical records and clinical evaluation they were categorized as: organic, nonorganic or mixed (both medical and behavioral). To assess nutritional status weight, length/height, and body mass index (BMI) were obtained and compared with WHO reference standards.

Results: Four hundred twenty-two children with mean age of 35 months (range: 3 to 156 months) were recruited. Fifty-three percent of feeding difficulties were nonorganic (behavioral), whereas the remaining $47 \%$ were caused by mixed factors. Organic factors were mostly related to gastroenterological disorders, food allergy or coexistence of more than one medical problem. Underweight (weight $<-2 z$-score) was observed in $18 \%$ of patients, stunted growth (length/height $<-2 z$-score) in $11 \%$, wasting (BMI $<-2 z$-score) in $15 \%$, and overweight (BMI > $+2 z$-score) in $1 \%$.

Conclusions: Our study showed that organic feeding difficulties coexisted with a behavioral component. The observed nutritional impairments in children with feeding difficulties indicate that there is a need for quick detection of these difficulties in order to provide appropriate support and help as soon as possible.
\end{abstract}

Key words: feeding difficulties, etiology, nutritional status, children.

\section{Introduction}

It is estimated that $20-40 \%$ of children experience some feeding difficulties [1, 2]. These problems are typically recognized in patients between 4 months and 6 years old [3]. Some children might experience feeding or eating difficulties due to motor problems [4]. Feeding difficulties are defined as patterns of oral consumption of solids and/or liquids which differ from the accepted norms. Feeding difficulty can manifest as partial or total food refusal, food selectivity (picky/fussy eating), food neophobia, and/or feeding disorders [2, 6]. It is an umbrella term encompassing all feeding problems, regardless of etiology or severity [2]. There exist several classifications of etiology of feeding difficulties, but none of them

\author{
Corresponding author: \\ Katarzyna M. Bąbik \\ Department of Pediatrics \\ Medical University of Warsaw \\ 61 Żwirki i Wigury St. \\ 02-127 Warszawa, Poland \\ E-mail: \\ katarzyna.babik@gmail.com
}


are perfect [7]. Feeding difficulty can be categorized as one of the three following factors: organic (medical), nonorganic (behavioral), and mixed (coexistence of organic and nonorganic factors) [8-11]. The results of published studies indicate that the majority of feeding problems are related to mixed causes [10,11], although the data about etiological factors are still limited.

During the first years of a child's life it is crucial to maintain a well-balanced diet in terms of quantity (energy) and quality (proper delivery of macronutrients, vitamins and trace elements). It ensures optimal growth and development of the child, and according to the theory of nutritional programming, simultaneously can determine the state of the health during adulthood [12]. Notably, feeding difficulties experienced by a child might lead to impairment of the nutritional status [2, 12, 13].

Our study aimed to assess etiological factors and nutritional status in children with feeding difficulties admitted to the multidisciplinary nutritional team.

\section{Material and methods}

\section{Design and study setting}

A cross-sectional study was conducted. Participants were recruited from among children admitted to the tertiary referral center for the assessment of feeding difficulties from September 2013 to April 2019.

\section{Study population}

Children with feeding difficulties who were admitted to the Department of Pediatrics were enrolled in the study. Feeding difficulties included feeding disorders (F98.2; ICD-10-CM)/avoidant-restrictive food intake disorder (ARFID; 307.59; F50.89, ICD-11) and food selectivity (picky/fussy

Table I. Characteristics of children with feeding difficulties

\begin{tabular}{|c|c|}
\hline \multicolumn{2}{|l|}{ Characteristic } \\
\hline Age [months], mean \pm SD & $35.46 \pm 32.70$ \\
\hline Length/height [z-score], mean \pm SD & $-0.39 \pm 1.23$ \\
\hline Weight [z-score], mean \pm SD & $-0.90 \pm 1.15^{*}$ \\
\hline BMI [z-score], mean \pm SD & $-0.96 \pm 1.17$ \\
\hline \multicolumn{2}{|l|}{ Sex, $n(\%)$} \\
\hline Boys & $234(55.45)$ \\
\hline Girls & $188(44.54)$ \\
\hline
\end{tabular}

Mean $\pm S D$ was calculated for $n=409, z$-scores for weight are available up to 10 years old. eating)/avoidant or restrictive eating (MB29.0; ICD-11). Feeding difficulties were diagnosed based on criteria defined in ICD-11. They were diagnosed by a minimum of two specialists via clinical interviews, in vivo observations of the mealtime, and analysis of video materials recorded at home during mealtimes.

\section{Data collection}

Part of the multidisciplinary nutritional team (psychologist, gastroenterologist and pediatrician) analyzed the patient's medical records and feeding history in order to categorized the cause of feeding difficulty as nonorganic, organic or mixed. If feeding difficulties were determined to be related to a medical condition, they were classified as organic [14]. When no medical problems were identified, then the feeding difficulty was categorized as being of nonorganic etiology [14]. Mixed etiologies were identified when there was coexistence of organic and nonorganic factors [15]. Nutritional status was assessed using anthropometric measurements (weight, length/ height) according to WHO protocols [16, 17]. Weight was measured with an electronic scale (Radwag WPT 6/15D or Radwag 60/150W). Length in children < 2 years was measured with infantometer (Seca 416). Height ( $>2$ years) was measured using a stadiometer (Seca 213).

\section{Data analysis}

Data were analyzed using IBM SPSS Statistics (version 24). Descriptive statistics were used to present the study population characteristics. Child's weight, length/height and body mass index (BMI) were converted into the standard deviation scores (z-scores) using the WHO Anthro ( $0-5$ years) and WHO AnthroPlus (5-19 years) software $[18,19]$. The data were interpreted based on WHO Global Database on Child Growth and Malnutrition recommendations [20, 21]. Underweight was defined as weight for age $<-2$ standard deviations (SD) of the WHO Child Growth Standards median, stunted growth as height for age $<-2$ SD of the WHO Child Growth Standards median, wasting as BMI <-2 SD of the WHO Child Growth Standards median, and overweight: weight for height $>+2$ SD of the WHO Child Growth Standards median [21].

\section{Results}

The data of four hundred and twenty-two children, referred to the hospital-based multidisciplinary feeding difficulties clinic in September 2013 - April 2019 in Warsaw, were analyzed. Characteristics of recruited population are included in Table I. 
The analyzed data showed that in 222 patients (53\%) feeding difficulties were related to behavioral factors only, whereas 200 patients (47\%) were affected by mixed factors: organic and behavioral. The most frequent organic causes related to the feeding difficulties were gastroenterological disorders (19.19\%), allergy (9.24\%), cardio-respiratory problems $(2.37 \%)$, neurological problems (1.89\%), nephrological problems (1.42\%), genetic disorders (1.42\%), endocrine problems (0.95\%), and laryngological problems (0.48\%). More than one medical problem was reported by 44 patients (10.43\%). Thirty-one patients were diagnosed with autism spectrum disorder, whereas 15 of those patients displayed feeding difficulties due to medical components such as allergy or gastroenterological disorders.

Most patients $(n=339)$ with feeding difficulties were between 0 and 5 years old. Only 83 patients were older than 5 years (Figure 1).

Underweight (weight $<-2$ z-score) was observed in 72 patients (17.60\%), stunted growth (length/height <-2 z-score) in 45 (10.66\%), wasting (BMI $<-2$ z-score) in 63 (14.93\%), and overweight (BMI > +2 z-score) in 5 (1.18\%). Both underweight and stunted growth were observed in 18 patients $(4.27 \%)$, underweight and wasting in 26 patients $(6.16 \%)$, stunted growth and wasting in 1 (0.24\%). Underweight, stunted growth and wasting were observed in 5 patients (1.18\%). Additionally, 5 patients were fed enterally during admission to the feeding clinic.

A summary of data on the nutritional status of both groups (organic and mixed) is presented in Table II.

There were no differences in means of z-score for weight, length/high and BMI between nonorganic and mixed causes. Anthropometric parameters (z-scores) for each factor are presented in Table III.

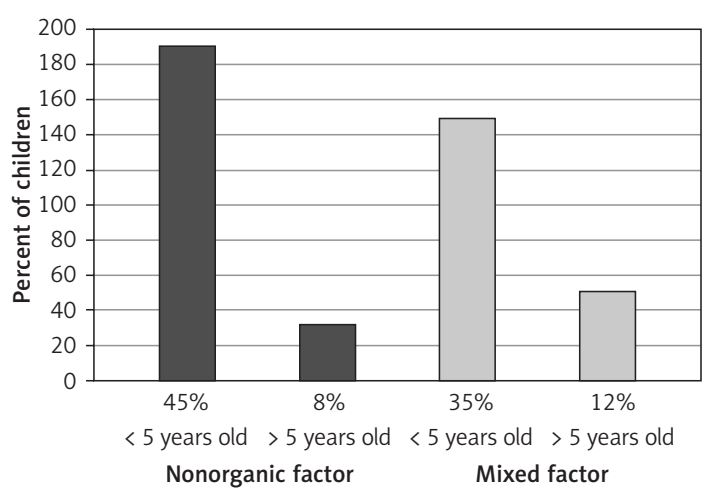

Figure 1. Etiology of feeding difficulties in children $<5$ years old and $>5$ years old

\section{Discussion}

The results of the current study showed that approximately half of the patients had isolated nonorganic feeding difficulties. The remaining children exhibited feeding problems due to mixed factors. Our results differ slightly from other published research $[8,10,11]$. We observed a higher proportion $(52 \%)$ of patients with isolated nonorganic feeding difficulties. In studies by Burklow et al. [11] and Budd et al. [10], nonorganic factors were observed in $12 \%$ and $10 \%$ of children respectively. We were unable to identify children with isolated organic factors, whereas other studies found that $15-26 \%$ of children had organic feeding difficulties $[10,11]$. This difference might be related to the fact that the current study was conducted in a tertiary referral center for the assessment of feeding difficulties. A majority of those patients were already diagnosed with feeding difficulties where organic factors had been ruled out or treated. Similarly to other publications, we found mixed etiology in a substantial proportion of patients $[8,10,11]$. Those results may suggest

Table II. Nutritional status in the pediatric population with feeding difficulties

\begin{tabular}{|lccccccc|}
\hline Child's age & \multicolumn{3}{c}{ Nonorganic factor } & & \multicolumn{2}{c|}{ Mixed factor } \\
\cline { 2 - 9 } & Underweight & $\begin{array}{c}\text { Stunted } \\
\text { growth }\end{array}$ & Wasting & Overweight & Underweight & $\begin{array}{c}\text { Stunted } \\
\text { growth }\end{array}$ & Wasting Overweight \\
\hline$<5$ years old, $n$ & 36 & 22 & 32 & 2 & 26 & 18 & 27 \\
\hline$>5$ years old, $n$ & 5 & 2 & 4 & 0 & 5 & 3 & 0 \\
\hline
\end{tabular}

Table III. Anthropometric parameters (z-scores) for nonorganic vs. mixed factors of feeding difficulties

\begin{tabular}{|lcccccc|}
\hline & \multicolumn{3}{c}{ Nonorganic factor } & & \multicolumn{2}{c|}{ Mixed factor } \\
\cline { 2 - 8 } & $\begin{array}{l}\text { Weight } \\
\text { z-score }\end{array}$ & $\begin{array}{c}\text { Length/height } \\
\text { z-score }\end{array}$ & $\begin{array}{c}\text { BMI } \\
\text { z-score }\end{array}$ & Weight & Length/height & B-score \\
z-score & z-score \\
\hline M & -0.97 & -0.41 & -1.02 & -0.82 & -0.36 & -0.89 \\
\hline SD & 1.11 & 1.23 & 1.10 & 1.20 & 1.23 & 1.24 \\
\hline Min; Max & $-3.85 ; 2.98$ & $-3.59 ; 2.96$ & $-3.78 ; 2.23$ & $-4.51 ; 3.19$ & $-4.12 ; 2.42$ & $-4.13 ; 3.50$ \\
\hline
\end{tabular}

$M-$ mean, SD - standard deviation, Min - minimum, Max-maximum. 
that medical symptoms are accompanied by behavioral food refusal, developed at a later point due to unpleasant experiences with feeding/eating during illness.

Problematic behaviors occurring during the mealtime may be maintained by environmental factors [22, 23]. In order to overcome a child's feeding difficulties, caregivers may try various strategies which reinforce those problems. Problematic behaviors would be maintained by negative and/or positive reinforcement. The mealtime context during the child's illness might be associated with pain or aversive experience, which may lead to engaging in food refusal and inappropriate behaviors (e.g. tantrums, pushing away spoon). Termination of the mealtime might be one of the responses of parents/caregivers [23]. A child learns that inappropriate mealtime behaviors produce escape from eating. A mealtime termination as a negative reinforcement of feeding problems results in increasingly inappropriate mealtime behavior in the future. Additionally, a child might engage in inappropriate mealtime behavior when it leads to access to tangibles, including highly preferred activities such as toys, movies, or attention. Continuation of that kind of dealing with problematic behaviors might increase them even more in the future. In this context, the positive reinforcement also maintains refusal behaviors $[22,24]$. It should be added that from the parent/ caregiver perspective stopping the child's inappropriate, difficult behavior is a powerful positive reinforcement for the termination of the mealtime. As a result, the parent/caregiver is likely, probably sooner than later, to end the mealtime. Thus, both the child's and the caregiver's behaviors are being reinforced, and the problem not only persists, but probably grows with time.

In our study we found that regardless of the etiology approximately $15 \%$ of children with feeding difficulties had $\mathrm{BMI}<-2$ z-score. We could not identify any publications assessing nutritional status in children with feeding difficulties regardless of the primary cause that would enable us to compare the data. Our results showed that detailed assessment of nutritional status should be one of the standards of care for children with feeding difficulties. The crucial part of the diagnostic and intervention process is cooperation between many specialists (pediatrician, gastroenterologist, psychologist, nutritionist, speech therapist) and the child's caregivers.

The strength of our study was a relatively large sample size. During the diagnostic process, we had access to the patients' detailed medical records and the data were complete. Moreover, the classification of feeding difficulties and etiology was done by at least two specialists: a medical doctor and a psychologist. Our study was conducted as a single-center study, which limits generalization of its results to other populations. However, given the limited number of treatment centers for feeding difficulties in our country, our recruited population might be representative. The cross-sectional design of our study does not prove causality. Another potential limitation of the study might be measurement error. However, all the measurements were done to minimize this problem; they were conducted using calibrated scales [25] always in the morning, following WHO protocols.

It is still unclear why some children with medical problems, despite receiving standardized therapy, develop behavioral problems. Additionally, the association of the parental BMI with the child's BMI and feeding difficulties and nutritional impairment should be explained. This issue needs to be addressed in further studies.

In conclusion, the current study focused on etiology and nutritional status in children with feeding difficulties. An isolated behavior component was present in more than half of our patients. Moreover, we observed that organic feeding difficulties always coexisted with a behavioral component. The nutritional impairment was detected in some patients regardless of etiology. Therefore it is crucial to start complex treatment in all patients with feeding difficulties as soon as possible to prevent potential health problems in the future.

\section{Conflict of interest}

The authors declare no conflict of interest.

\section{References}

1. Bentovim A. The clinical approach to feeding disorders of childhood. J Psychosom Res 1970; 14: 267-76.

2. Kerzner B, Milano K, MacLean WC Jr, Berall G, Stuart S, Chatoor I. A practical approach to classifying and managing feeding difficulties. Pediatrics 2015; 135: 344-53.

3. Aldridge VK, Dovey TM, Martin Cl, Meyer C. Identifying clinically relevant feeding problems and disorders. J Child Health Care 2010; 14: 261-70.

4. Gieysztor EZ, Choińska AM, Paprocka-Borowicz M. Persistence of primitive reflexes and associated motor problems in healthy preschool children. Arch Med Sci 2018; 14: 167-73.

5. Linscheid TR. Behavioral treatments for pediatric feeding disorders. Behav Modif 2006; 30: 6-23.

6. Yang HR. How to approach feeding difficulties in young children. Korean J Pediatr 2017; 60: 379-84.

7. Goday PS, Huh SY, Silverman A, et al. Pediatric feeding disorder: consensus definition and conceptual framework. J Pediatr Gastroenterol Nutr 2019; 68: 124-9.

8. Rommel N, De Meyer AM, Feenstra L, Veereman-Wauters G. The complexity of feeding problems in 700 infants and young children presenting to a tertiary care institution. J Pediatr Gastroenterol Nutr 2003; 37: 75-84.

9. Williams KE, Riegel K, Kerwin ML. Feeding disorder of infancy or early childhood: how often is it seen in feeding programs? Childrens Health Care 2009; 38: 123-36. 
10. Budd KS, McGraw TE, Farbisz R, et al. Psychosocial concomitants of children's feeding disorders. J Pediatr Psychol 1992; 17: 81-94.

11. Burklow KA, Phelps AN, Schultz JR, McConnell K, Rudolph C. Classifying complex pediatric feeding disorders. J Pediatr Gastroenterol Nutr 1998; 27: 143-7.

12. Rybak A. Organic and nonorganic feeding disorders. Ann Nutr Metab 2015; 66 Suppl 5: 16-22.

13. Sullivan PB, Juszczak E, Lambert BR, Rose M, FordAdams ME, Johnson A. Impact of feeding problems on nutritional intake and growth: Oxford Feeding Study II. Dev Med Child Neurol 2002; 44: 461-7.

14. Kedesdy JH, Budd KS. Childhood Feeding Disorders: Biobehavioral Assessment and Intervention. Baltimore, MD: Paul H. Brookes Publishing Company; 1998.

15. Iwata BA, Riordan MM, Wohl MK, W. FJ. Pediatric feeding disorders: behavioral analysis and treatment. In: Accardo PJ (ed.). Failure to thrive in infancy and early childhood: a multidisciplinary approach. Baltimore, MD: University Park Press; 1982, pp. 297-329.

16. Onyango AW, dO, W. Training Course on Child Growth Assessment. Geneva; 2008.

17. de Onis M, Onyango AW, Van den Broeck J, Chumlea WC, Martorell R. Measurement and standardization protocols for anthropometry used in the construction of a new international growth reference. Food Nutr Bull 2004; 25 (1 Suppl): S27-36.

18. WHO. WHO Anthro Survey Analyser: Software for analysing survey anthropometric data for children under 5 years of age. Built-in software edition. 1.0 edn. Geneva: World Health Organization; 2018.

19. WHO. WHO AnthroPlus for personal computers Manual: Software for assessing growth of the world's children and adolescents. Geneva: World Health Organization; 2009.

20. WHO. Nutrition Landscape Information System (NLIS) country profile indicators: interpretation guide. 2010. https://www.who.int/nutrition/nlis_interpretation guide.pdf [Accessed: 20.11.2019].

21. WHO. Training Course on Child Growth Assessment. Geneva: World Health Organization; 2008.

22. Bąbik K, Suchowierska M, Ostaszewski P. Implementation of behavioral therapy as a primary intervention for children and teenagers to increase solids and liquids acceptance: systematic review of the empirical researches. Postępy Nauk Medycznych 2015; 7: 524-534.

23. Piazza CC, Fisher WW, Brown KA, et al. Functional analysis of inappropriate mealtime behaviors. J Appl Behav Anal 2003; 36: 187-204.

24. Bąbik K. Trudności związane z karmieniem u dzieci selektywne jedzenie, odmowa jedzenia, zaburzenia karmienia. Pol J Nutr 2016; 2: 67-71.

25. Ulijaszek SJ, Kerr DA. Anthropometric measurement error and the assessment of nutritional status. Br J Nutr 1999; 82: 165-77. 